\title{
Post coronary artery bypass graft surgery thyroid storm-A rare endocrinological emergency
}

Keywords: stenosis, tachycardic, thyrotoxicosis, surgery

\section{Case report}

Altered mental status (AMS) is a thought-provoking symptom frequently encountered post surgically in inpatient hospital setting. The differential diagnosis is extensive and arrays from simple delirium to new onset cerebrovascular event. ${ }^{1}$ AMS work up ranges from basic history and physical exam to advanced radiological tests like gadolinium enhanced MR. Herein we report a rare case of thyroid storm post coronary artery bypass graft (CABG) surgery in a patient with no past medical history of thyroid disorder. A 58-year-old female with past medical history of diabetes mellitus and depression presented with chest pain and dyspnea. Her electrocardiogram showed ST segment elevation in the anteroseptal leads. An emergent primary PCI was done which demonstrated tight proximal stenosis in the LAD, moderate to severe stenosis in left circumflex artery and moderate stenosis in RCA. A CABG surgery was planned for the next day and she had a smooth intraoperative and immediate post-operative course. However on day 3 , she was febrile to $103 \mathrm{~F}$, tachycardic to 134 beats per minute, hypertensive with systolic pressure in 200's and had sudden deterioration in mental status. Comprehensive work up for fever and AMS including blood cultures, chest X-ray, urine analysis and culture, peripheral blood smear for malaria, serology for leptospirosis, streptococcus and mycoplasma, serum ammonia, lumbar puncture, EEG and CT brain, was unremarkable. She was treated with broad spectrum antibiotics, anti-platelets, beta blockers and nitroglycerine drip; however she remained obtunded, febrile and tachycardic. Thyroid profile was obtained on post-operative day 5 and revealed thyroid stimulating hormone $(\mathrm{TSH})<0.05 \mathrm{uU} / \mathrm{ml}$, thyroxine (T4) $>180.5 \mathrm{nmol} / 1$ and triiodothyronine (T3) $2.69 \mathrm{nmol} / \mathrm{l}$. Thyroid sonogram revealed multiple enlarged hyper vascular thyroid nodules. She was started on stress dose steroids, methimazole and continued on beta blocker. Within the next 3 days her heart rate, blood pressure, fever and mental status improved. She was finally discharged home on oral methimazole and tapering dose of steroids with follow up in endocrinology clinic in 1 month on day 10 post operatively. Thyroid storm is a rare, life-threatening endocrinological emergency with 2030 percent. Mortality if not treated promptly and is characterized by severe clinical manifestations of thyrotoxicosis. It may be triggered by an acute event such as thyroid or nonthyroidal surgery, trauma, infection, an acute iodine load, or parturition. It is unclear why certain factors result in the development of thyroid storm. Hypotheses include a rapid rate of increase in serum thyroid hormone levels, increased responsiveness to catecholamines, or enhanced cellular responses to thyroid hormone., ${ }^{2,3}$ The degree of thyroid hormone excess (elevation of T4 and T3) and suppression of TSH typically is not more profound than that seen in patients with uncomplicated thyrotoxicosis. ${ }^{4}$ In this report, we have described a case of thyroid storm after CABG in a 58 -year-old female patient with no known history of either thyroid or coronary artery disease. Thyroid storm was diagnosed according to the Burch-Wartofsky criteria. ${ }^{5}$ This set of diagnostic criteria assesses the likelihood of thyroid storm by scoring clinical features such as body temperature, central nervous system effects, gastrointestinal or
Volume 12 Issue I - 2019

\author{
Usman Khan, Muhammad Bajwa \\ Department of Medicine, Division of Nephrology, University of \\ Oklahoma Health Sciences Center, USA
}

Correspondence: Usman Khan, Department of Medicine, Division of Nephrology, University of Oklahoma Health Sciences Center, USA, Oklahoma, USA, Tel 4056974750 Email Usman-Khan@ouhsc.edu

Received: December 10, 2018 | Published: January 14, 2019

hepatic dysfunction, heart rate, heart failure, atrial fibrillation, and precipitant history. The scale ranges from 0 to 140 points, with a score greater than 60 being highly compatible with thyroid storm. The final diagnosis was based not only on this score but also on thyroid function tests and response to therapy. Persistent hypertension, new onset atrial fibrillation, absence of muscle rigidity and negative blood cultures, CT head and EEG differentiated thyroid storm from other causes of AMS, like sepsis, hypoxemia, neuroleptic malignant syndrome, stroke and hypoglycemia.

Thyroid storm post CABG has been previously reported in literature. ${ }^{6-8}$ Our case has multiple features in common with the previous reports, such as female sex, few risk factors for coronary artery disease, minimal preoperative evidence of thyrotoxicosis, and postoperative storm complicated by AMS and a prompt response to thyroid suppression therapy. The recognized triggers for thyroid storm were surgery and iodine load in our case. Standard therapy for thyroid storm includes thyroid blocking agents such as thioamide, steroids, beta blockers and iodine. Thioamide such as methimazole and propylthiouracil blocks the synthesis of thyroid hormone in the thyroid. Iodine therapy suppresses release of thyroid hormone through the Wolff-Chaikoff effect. Hydrocortisone therapy blocks peripheral conversion of thyroxine to triiodothyronine. In hemodynamically stable patients, beta blockade is useful to counteract the increased adrenergic tone observed in thyroid storm. It is important to note that thyrotoxicosis may be associated with severe coronary spasm that may mimic STEMI like picture. ${ }^{9}$ In summary, we wanted to alert clinician of thyroid storm which is a rare endocrinological emergency with 30 percent mortality and it can be precipitated by iodine load and CABG surgery in completely asymptomatic undiagnosed hyperthyroid patients. Although rare, this postoperative complication is important because it is life-threatening and yet eminently treatable.

\section{Acknowledgments}

None.

\section{Conflicts of interest}

Author declares that there are no conflicts of interest.

\section{References}

1. Francis J, Martin D, Kapoor WN. A prospective study of delirium in hospitalized elderly. Jama. 1990;23;263(8):1097-1101. 
2. Sarlis NJ, Gourgiotis L. Thyroid Emergencies. Rev Endocr Metab Disord. 2003;4(2):129-136.

3. Tietgens ST, Leinung MC. Thyroid storm. Med Clin North Am. 1995;79(1):169-184.

4. Carroll R, Matfin G. Endocrine and metabolic emergencies: thyroid storm. Ther Adv Endocrinol Metab. 2010;1(3):139-145.

5. Burch HB, Wartofsky L. Life-threatening thyrotoxicosis. Thyroid storm Endocrinol Metab Clin North Am. 1993;22(2):263-277.
6. Bish LT, Bavaria JE, Augoustides J. Thyroid storm after coronary artery bypass grafting. J Thorac Cardiovasc Surg. 2010;140(5):e67-e69.

7. Lee SM, Jung TS, Hahm JR, et al. Thyrotoxicosis with coronary spasm that required coronary artery bypass surgery. Intern Med. 2007;46(23):1915-1918.

8. Dhillon A, Manocha D, Kahlon A, et al. Post coronary artery bypass graft (CABG) surgery thyroid storm: a near miss. 2017.

9. Choi YH, Chung JH, Bae SW, et al. Severe coronary artery spasm can be associated with hyperthyroidism. Coron Artery Dis. 2005;16(3):135-139. 